\title{
Ontology Metadata Vocabulary and Applications
}

\author{
Jens Hartmann ${ }^{1}$, Raúl Palma ${ }^{2}$, York Sure ${ }^{1}$, M. Carmen Suárez-Figueroa ${ }^{2}$, \\ Peter Haase $^{1}$, Asunción Gómez-Pérez ${ }^{2}$, and Rudi Studer ${ }^{1}$ \\ 1 Institute AIFB, University of Karlsruhe, Germany \\ 2 Ontology Engineering Group, Laboratorio de Inteligencia Artificial, \\ Facultad de Informática, \\ Universidad Politécnica de Madrid, Spain
}

\begin{abstract}
Ontologies have seen quite an enormous development and application in many domains within the last years, especially in the context of the next web generation, the Semantic Web. Besides the work of countless researchers across the world, industry starts developing ontologies to support their daily operative business. Currently, most ontologies exist in pure form without any additional information, e.g. authorship information, such as provided by Dublin Core for text documents. This burden makes it difficult for academia and industry e.g. to identify, find and apply - basically meaning to reuse - ontologies effectively and efficiently. Our contribution consists of (i) a proposal for a metadata standard, so called Ontology Metadata Vocabulary (OMV) which is based on discussions in the EU IST thematic network of excellence Knowledge Web! and (ii) two complementary reference implementations which show the benefit of such a standard in decentralized and centralized scenarios, i.e. the Oyster P2P system and the Onthology metadata portal.
\end{abstract}

\section{Introduction}

Ontologies are commonly used for a shared means of communication between computers and between humans and computers. To reach this aim, ontologies should be represented, described, exchanged, shared and accessed based on open standards. Consider, as an example, the W3C standardized web ontology language OWL [10]. Currently, most ontologies exist in pure form without any additional information, e.g. authorship information, such as provided by Dublin Core for text documents. This burden makes it difficult for academia and industry to identify and apply - basically meaning to reuse ontologies effectively and efficiently. Metadata is meant as machine processable information for the Wet? It is a systematic method for describing information resources, helps to improve their accessibility and gives other useful resource information to support their maintenance (e.g. to find data sets, to determine whether the data set is appropriate for a certain use, etc.). Thus, one key purpose of metadata is to facilitate and improve the retrieval of information.

Taking into account that ontology sharing and reuse is quite often difficult for academia and industry and the main features of metadata, they could be used for

\footnotetext{
1 http: //knowledgeweb.semanticweb.org/

2 http://www.w3.org/Metadata/
} 
describing ontologies (the outcome of this would be ontology metadata) for sharing, exchanging and reusing them in a most efficient way. To achieve this goal, it is necessary to agree on a standard for ontology metadata, that is a common set of terms and definitions describing ontologies, so called metadata vocabulary. Then, implementing such a vocabulary will increase the value of ontologies by facilitating ontology sharing and reusing through time and space. If ontologies are described using ontology metadata standards, an appropriate technology infrastructure is required. For example, tools and metadata repositories, compatible to the ontology metadata standards, must be developed. These tools and repositories can as a consequence e.g. support the creation, maintenance and distribution of ontology metadata.

Our contribution consists of (i) a proposal for a metadata standard for capturing properties ontologies supporting their reuse, so called Ontology Metadata Vocabulary (OMV), which is based on discussions and agreement in the EU IST thematic network of excellence Knowledge Web and (ii) two complementary reference implementations which show the benefit of such a standard in decentralized and centralized scenarios, i.e. the P2P system Oyster and the metadata portal Onthology. This paper is organized as follows: section 1 provides the introduction. The developed metadata vocabulary is given in section 2 introduced by the main requirements. The P2P system Oyster and the portal Onthology are described in section 3 . In section 4 we provide related work, conclude and present future work.

\section{Ontology Metadata Vocabulary}

\subsection{Requirements}

As an initial step towards a standardized vocabulary, we analysed requirements for ontology metadata. Several aspects are similar to other metadata standards, like Dublin Core. However, important differences like the conceptual models (semantics) behind ontologies require a detailed analysis and require a different representation of metadata about ontologies. In a nutshell, an ontology normally reflects the (i) conceptualization from persons about a specific task or domain which then is (ii) realised by an ontology engineering process [12].

As a result, the main identified requirements are the following:

- Accessibility: Metadata, especially about ontologies, must be accessible and processable for humans and machines.

- Usability: a majority of users should be able to apply metadata easily.

- Reuse of Ontologies: As ontologies are a core technology for the Semantic Web, its metadata should reflect key issues of the Semantic Web as well. In particular reuse and sharing of knowledge.

- Conceptualisation vs. Realisation: Metadata must reflect (and also distinguish between) a semantic conceptualisation and its particular realisation as a concrete ontology document.

- Interoperability: Metadata should be interoperable and conform to the major representation languages currently being used for Semantic Web applications. Indeed, this means that a metadata vocabulary should be representable e.g. in F-Logic and OWL as well. 
- Documentary: Documentary aspects of metadata like information about technical, statistical, accessibility, management information, etc. should be provided.

- Extensibility: Reflecting special user needs, it is required that beyond such standard metadata facts can be added and extended easily.

- Expressiveness: Metadata must be expressive enough to represent all desired aspects, as presented above.

The main aspects are Conceptualisation vs. Realisation and Reuse of Ontologies which should be reflected by any ontology metadata. Already now, it is possible to capture several technical properties of ontologies, like used syntax or number of classes, almost automatically like realised by [3] for example. Besides technical properties which are obviously relevant, there is a strong demand for representing conceptual metadata, like authorship information, categorizations or underlying methodologies. As consequence, representing these issues by a vocabulary requires an expressive language for the metadata itself which makes it impossible to reuse any existing metadata schema.

\subsection{Conceptualisation vs. Realisation}

OMV distinguishes between an ontology base and an ontology document. This separation is based on following observation: any existing ontology document has some kind of core idea (conceptualisation) behind. From an ontology engineering perspective, initially a person develops such core idea of what should be modeled (and maybe how) in his mind. Further, this initial conceptualisation might be discussed with other persons and after all, an ontology will be realized using an ontology editor and stored in a specific format. Over time, there might be created several realizations of this initial cconceptualisation in many different formats, e.g. in $\mathrm{RDF}(\mathrm{S})$ [1] or OWL [10].

Therefore we distinguish between an ontology base and an ontology document:

- [Ontology Base]: An Ontology Base (OB) represents the abstract or core idea of an ontology, so called conceptualisation. It describes the core properties of an ontology, independent from any implementation details. For a general illustration of the relationship of an $\mathrm{OB}$ and $\mathrm{OD}$, we refer to figure 1 .

- [Ontology Document]: An Ontology Document $(O D)$ represents a specific realization of an ontology base. Therefore, it describes properties of an ontology that are related to the realization or implementation.

The distinction between an OB and OD leads to an efficient mechanism, e.g. for tracking several versions and evolvements of ontologies as well as for different representations of one knowledge model (conceptualisation) in different languages. In particular, such an ontology base can be seen as representation of the conceptual model

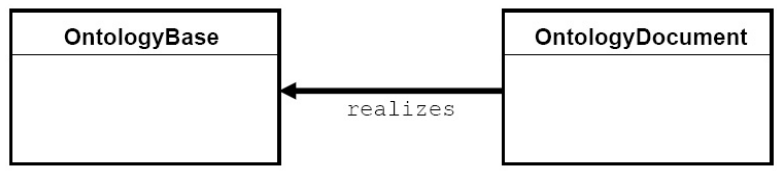

Fig. 1. Relationship between $\mathrm{OB}$ and $\mathrm{OD}$ 
behind an ontology. Technically, an ontology base and an ontology document are modeled as two separate classes, with the relation reali zes from the ontology document to the ontology base. This means that there may be many possible ontology documents for one ontology base, but one ontology document can only realize one ontology base.

Normally, an OD should not be able to exist without a corresponding OB. However, for practical reasons, we allow the existence of each class independently of each other. Hence, we cannot assume that every existing ontology will be annotated by its original author who might create an $\mathrm{OB}$ for his ontology. However, automatically extracting syntactical properties of an existing ontology is quite simple. Then, such minimal $O D$ would exist without a concrete $\mathrm{OB}$.

The main classes and properties of the OMV ontology are illustrated in figure 2 Please notice, that not all classes and properties are included. It is only to demonstrate the main idea behind OMV. The complete ontology is described in [6] and is available for download in several ontology formats 3 .

It should be noticed that there exist several properties within OB and OD which look similar at first. However, they have different meanings and semantics. Exemplary, think of an ontology engineer A developing an ontology in $\mathrm{RDF}(\mathrm{S})$ syntax and annotating it with OMV. Then, the properties of an OB and OD individual are quite similar. Exemplary, both would have the same party as creator and so on. However, over time, there might be another engineer $B$ with similar needs according to the OB from $A$. Hence, $B$ reuses the $\mathrm{OB}$ from $\mathrm{A}$ and only creates a new $\mathrm{OD}$, e.g. realizing the $\mathrm{OB}$ in OWL instead of $\mathrm{RDF}(\mathrm{S})$. As a result, a new OD would be created for this ontology and most of the properties are different now.

\subsection{Properties to Support Reuse in OMV}

As mentioned above, the OMV models the two main classes OB and OD for representing core information about ontologies. However, additional classes are required to represent and support the reuse of ontologies by such metadata vocabulary, especially in the context of the Semantic Web. Hence, we modeled, as shown in figure 2, further classes and properties representing environmental information and relations. We will briefly discuss these classes in the following. In typical ontology engineering mainly a Person (or multiple) or an Organisation as a whole are developing ontologies. We group these two classes under the generic class Party by a subclass-of relation. A Party can create, contribute and review an OntologyBase resp. an ontologyDocument. We here distinguish between the development of an OB and OD. Further, tools such as ontology editors can be referred to by the class OntologyEngineeringTool which itself can be developedBy a Party. The different existing syntactical representations and ontology languages are representable by Ontologysyntax and OntologyLanguage. OMV further consists of the class KM-Method make explicit the methodology (or methodologies) used during engineering. Ontologies might be categorized by different types of ontologies, exemplary think of domain, lingusitic or upper-level ontologies. Those types can be modeled by the class OntologyType. For industry it might be relevant to propose usage licenses

${ }^{3}$ OMV representations are available at http: / / ontoware. org/projects/omv/ 


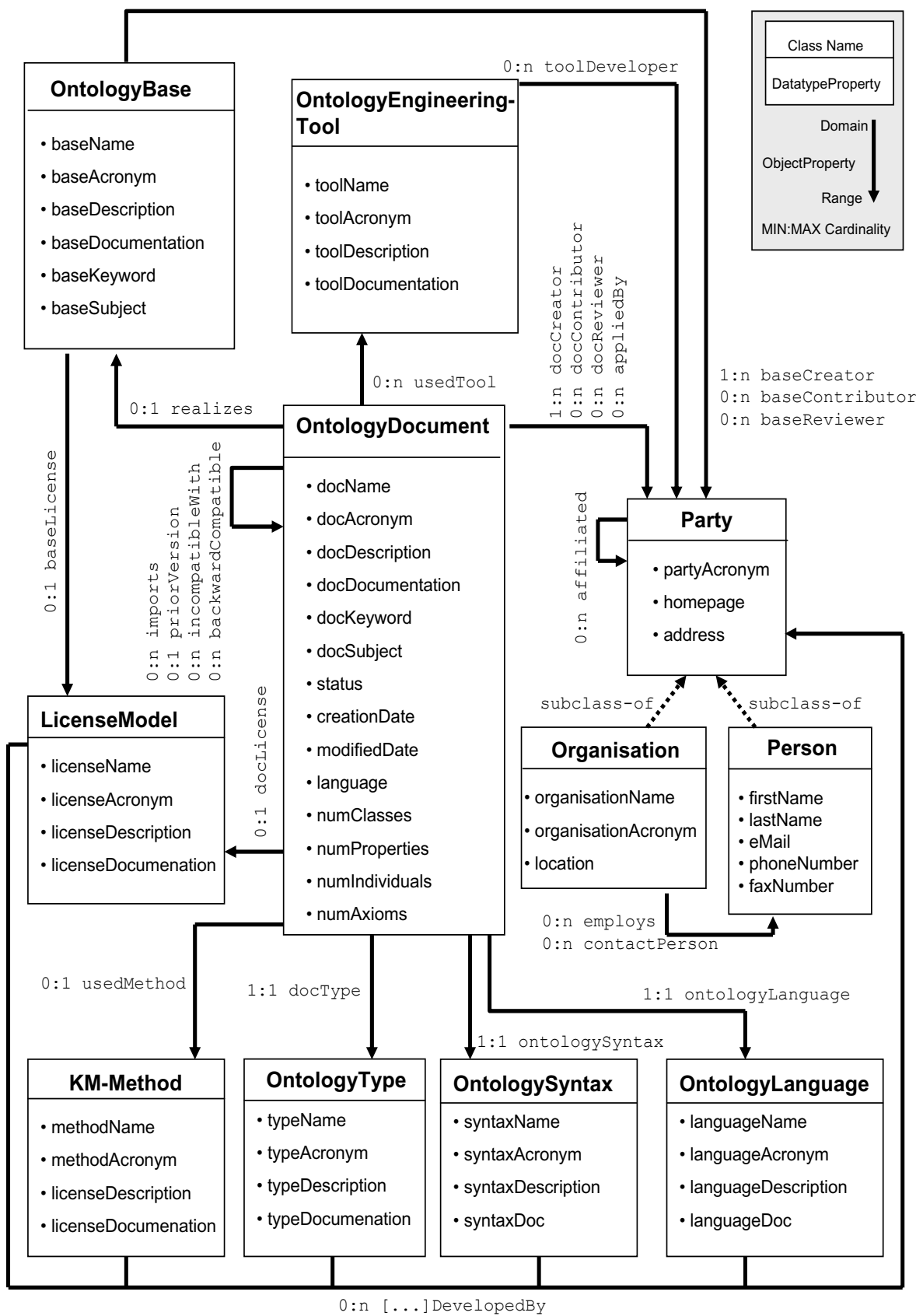

Fig. 2. General OMV overview 
which can be realized by the class LicenseModel. So, that each OntologyBase or OntologyDocument is related to a pre-defined LicenseModel.

The presented OMV tries to model as much information about ontologies and the important aspects for ontology reuse as possible and at the same time intends to stay as simple as possible.

\section{Applications for OMV}

We now present running applications based on the proposed OMV. In detail, we present two complementary applications, namely the decentralised $\mathrm{P} 2 \mathrm{P}$ system Oyster and the centralised metadata portal Onthology. Both applications have in common that they support single users and communities of users in indentifying, reusing and providing ontology metadata. As a consequence, they support the core idea of the Semantic Web and help to increase the applicability of ontologies.

In general, the two tools differ in their usage perspective and are appropriate for different tasks. However, as we will see, only the combined application of both tools will offer users the full potential of ontology metadata management.

\subsection{Oyster - A Peer-to-Peer System for Sharing Ontologies}

Overview. Oystert is a Java-based system that exploits semantic web techniques in order to provide an innovative and useful solution for exchanging and reusing ontologies. For this purpose, Oyster provides facilities for managing, searching and sharing ontology metadata in a P2P network, thereby implementing the OMV proposal for the standard set of ontology metadata.

Oyster offers a user driven approach where each peer has its own local repository of ontology metadata and also has access to the information of others repositories, thus creating a virtual decentralized Ontology repository. The Oyster client on its own (e.g. disconnected from the $\mathrm{P} 2 \mathrm{P}$ network) will already provide added value to its users as it will give developers an overview and search facilities of his/her own ontology metadata stored in its local repository. The goal is a decentralized knowledge sharing environment using Semantic Web technologies that allows developers to easily share ontology documents.

Functionalities. The Oyster system has been implemented as an instance of the Swapster system architecture 5 . It uses ontologies extensively in order to provide some of its main functions: importing data, formulating queries and processing answers.

Creating and importing metadata: Oyster enables users to create metadata about ontologies manually, as well as to import ontology files and to automatically extract the ontology metadata available, letting the user to fill in missing values. The ontology metadata entries are aligned and formally represented according to two ontologies: (1) the OMV ontology6, (2) a topic hierarchy (i.e. the DMOZ topic hierarchy), which describes specific categories of subjects to define the domain of the ontology.

\footnotetext{
4 http: / / oyster.ontoware.org/

5 http: / / swap.semanticweb.org/

6 http://omv.ontoware.org/2005/05/ontology
} 
Formulating queries: A user can search for ontologies using simple keyword searches, or using more advanced, semantic searches. Here, queries are formulated in terms of these two ontologies. This means queries can refer to fields like name, acronym, ontology language, etc. or queries may refer to specific topic terms.

Routing queries: A user may query a single specific peer (e.g. their own computer, because they can have many ontologies stored locally and finding the right one for a specific task can be time consuming, or users may want to query another peer in particular because this peer is a known big provider of information), or a specific set of peers (e.g. all the member of a specific organization), or the entire network of peers (e.g. when the user has no idea where to search), in which case queries are routed automatically in the network.

Processing results: Finally, results matching a query are presented in a result list. The answer of a query might be very large, and contain many duplicates due to the distributed nature and potentially large size of the P2P network. Such duplicates might not be exactly copies because the semi structured nature of the metadata, so the ontologies are used again to measure the semantic similarity between different answers and to remove apparent duplicates. As proposed by the ontology metadata standard, all the different realizations of an ontology (ontology documents) can be grouped by the same ontology base to give a more organized view of the results.

\subsection{Onthology - A Central Ontology Metadata Portal}

As the importance of metadata increases with the number of existing ontologies, the storage and access to it becomes important as well. There exist mainly two kinds of storage facilities for ontology metadata. We present the conceptual design of a centralised ontology metadata portal and its implementation, so-called Onthology meaning an anthology of ontologies?.

Actors. A main goal of a centralised metadata portal is to act as large evidence storage of metadata resp. their related ontologies to assure access, reuse and sharing, in the sense of the Semantic Web. We identified several different user roles for Onthology: The visitor is an anonymous user, he is allowed to browse the public content of the portal. A Visitor can become a user by completing an application form on the website. In order to avoid unnecessary administrative work, a User is added automatically to the membership database. Users can customize their portal, e.g. the content of their startpage or their bookmarks. If a user wants to add metadata to the portal, this submission has to be reviewed before it is published. Onthology works with a review process in order to ensure the quality of the metadata. Reviewers check the new submissions before it is published. The technical administrator is responsible for any other task mainly the maintenance of the portal.

Functionalities. Functionalities of Onthology can be separated into two groups based on the usage. Indeed, basic functionalities are provided to every user who accesses the repository and sophisticated functionalities for reviewers and administrators. The main operations a user can perform on the repository are the following.

\footnotetext{
${ }^{7}$ http: //www. onthology.org/
} 
- Search: Query and browse the repository

- Submit: Provide new metadata

- Export: Download (parts of) the repository.

The search and export can be performed by any visitor without being registered to the repository. Since providing new metadata is based on a certain community confidence, a visitor has to register at the repository to be become a registered user.

Architecture. A metadata portal mainly consists of a large data repository in which metadata can be stored. Exemplary, Sesam 8 or KAON9 can be used as back-end metadata repository. Furthermore, access and in particular the managament of the repository must be guaranteed, too. Therefore, Onthology is based on SEAL, the AIFB conceptual architecture for building SEmantic portALs. In SEAL ontologies are key elements for managing community web sites and web portals. They support queries to multiple sources, but beyond that also intensive use of the schema information itself to allow for automatic generation of navigational views such as navigation hierarchies that appear as has-part-trees or has-subtopic trees in the ontology. In addition to that mixed ontology and content-based presentation is supported. Further information can be found at [7].

\subsection{Discussion}

Both presented applications are covering a variety of different tasks. Indeed, users who wants to store metadata individually similar to managing his personal favorite song list, a repository is required to which a user has full access and can perfom any operation (e.g. create, edit or delete metadata) without any consequences to other users. Exemplary, users from academia or industry might use a personal repository for a taskdependant investigation or ontology engineers, might use it during their ontology development process to capture information about different ontology versions. We argue, that a decentralised system is the technique of choice, since it allows the maximum of individuality while it still ensures exchange with other users.

Centralised systems allow to reflect long-term community processes in which some ontologies become well accepted for a domain or community and others become less important. Such well accepted ontologies and in particular their metadata need to be stored in a central metadata portal which can be accessed easily by a large number of users whereby the management procedures are well defined. Obviously, personal repositories are quite limited from this perspective.

Actually, the Oyster system and Onthology are not necessarily two completely separated repositories. Indeed, they are interconnected and they exchange metadata between each other. We are currently supporting the access of metadata stored in Onthology from any Oyster peer. However, accessing metadata in Onthology stored on Oyster peers is future work and requires more conceptual work, because the stored metadata within Onthology are based on a certain level of confidence among a community.

\footnotetext{
${ }^{8}$ http: //www.openrdf.org/

9 http: / / kaon. semanticweb.org/
} 
The benefit of connecting both systems lies mainly in the simple use of existing ontology metadata information within Oyster. So, while users are applying or even developing their own ontologies they can manage their own metadata along with other existing metadata in one application (in Oyster). If some metadata entries from Oyster have reached a certain confidence, an import into Onthology can be performed easily. In combination, both systems ensure efficient and effective ontology metadata management for various use cases.

\section{Related Work and Conclusion}

We will briefly mention related metadata standards, including in particular those ones relevant for the Semantic Web. The Dublin Core (DC) metadata standard [2] is a simple yet effective element set for describing a wide range of networked resources. It includes two levels: Simple and Qualified. Simple DC comprises fifteen elements; Qualified DC includes an additional element as well as a group of element refinements (or qualifiers) that refine the semantics of the elements in ways that may be useful in resource discovery. FOAF [5], or "Friend Of A Friend", provides a way to create machine-readable Web homepages for people (their interests, relationships and activities), groups, companies and other kinds of things. To achieve this, FOAF project use the "FOAF vocabulary" to provide a collection of basic terms that can be used in these Web pages. The initial focus of FOAF has been on the description of people. The Semantic Web search engine SWOOGLE [3] makes use of particularly those metadata which can be extracted automatically. Our approach includes and extends this metadata vocabulary. Ideally, future versions of SWOOGLE would also take into account the additional vocabulary defined in OMV. There exist some similar approaches to our proposed solution to share ontologies, but in general they are limited in scope. E.g. the DAML ontology library [9] provides a catalog of DAML ontologies that can be browsed by different properties. The FIPA ontology service [11] defines an agent wrapper of open knowledge base connectivity. Finally we mention the SchemaWeb Directory [4] that is a repository for RDF schemas expressed in RDFS, OWL and DAML+OIL. While the goal of SchemaWeb is similar to that of Onthology, its metadata ontology is less comprehensive.

The term ontology base is used in different context in DOGMA[8]: A DOGMA ontology consists of an ontology base that holds sets of intuitive context-specific conceptual relations and a layer of "relatively generic" ontological commitments that hold the domain rules. In contrast, in our work we use the term ontology base to represent the conceptualisation of an ontology.

To conclude, reusing existing ontologies is a key issue for sharing knowledge on the Semantic Web. Our contribution aims at facilitating reuse of ontologies which are previously unknown for ontology developers by providing an Ontology Metadata Vocabulary (OMV) and two prototypical applications for decentralized (Oyster) and centralized (Onthology) sharing of ontology metadata based on OMV.

OMV has been proposed and discussed in the industry area of the EU thematic network of excellence Knowledge Web (KWeb). Next steps include the standardization of OMV in a wider scope by particularly including non-KWeb parties in this process, 
followed by a close cooperation with tool providers for ontology engineering environments and applications providers for e.g. ontology based search engines to enhance their tools with support for OMV. The agreement and application of a standard on a global level will greatly facilitate the reuse of ontologies for all participating parties.

Acknowledgments. Research reported in this paper has been partially financed by EU in the IST project Knowledge Web (FP6-507482).

\section{References}

1. D. Brickley and R. V. Guha. RDF Vocabulary Description Language 1.0: RDF Schema. W3C Rec. 10 February 2004, 2004. available at http: / / www .w3 . org/TR/rdf-schema/.

2. Dublin Core. http: / / dublincore.org/

3. Li Ding et al. Swoogle: A search and metadata engine for the semantic web. In In Proceedings of the Thirteenth ACM Conference on Information and Knowledge Management, pages 58-61, November 2004.

4. SchemaWeb directory. http://www. schemaweb. info/

5. FOAF. http: //www. foaf-project.org/

6. J. Hartmann and R. Palma. OMV - Ontology Metadata Vocabulary for the Semantic Web, 2005. v. 1.0, available at http: / / omv. ontoware.org/

7. J. Hartmann and Y. Sure. An infrastructure for scalable, reliable semantic portals. IEEE Intelligent Systems, 19(3):58-65, May/June 2004.

8. Spyns Peter, Meersman Robert, and Jarrar Mustafa. Data modelling versus ontological engineering. In SIGMOD Record Special Issue on Semantic Web, Database Management and Information Systems, volume 31, pages 7-12, December 2002.

9. DAML Ontology Repository. http: / /www. daml.org/ontologies/

10. M. K. Smith, C. Welty, and D. McGuinness. OWL Web Ontology Language Guide, 2004. W3C Rec. 10 February 2004, available at http: / /www.w3 . org/TR/owl-guide/

11. H. Suguri et al. Implementation of fipa ontology service. In Proc. of the Workshop on Ontologies in Agent Systems, 5th Int. Conf. on Autonomous Agents Montreal, Canada, 2001.

12. C. Tempich, H. S. Pinto, Y. Sure, and S. Staab. An argumentation ontology for distributed, loosely-controlled and evolving engineering processes of ontologies (diligent). In A. GezPez and J. Euzenat, editors, 2nd European Semantic Web Conference, ESWC 2005, volume 3532 of LNCS, pages 241-256, Heraklion, Crete, Greece, MAY 2005. Springer. 